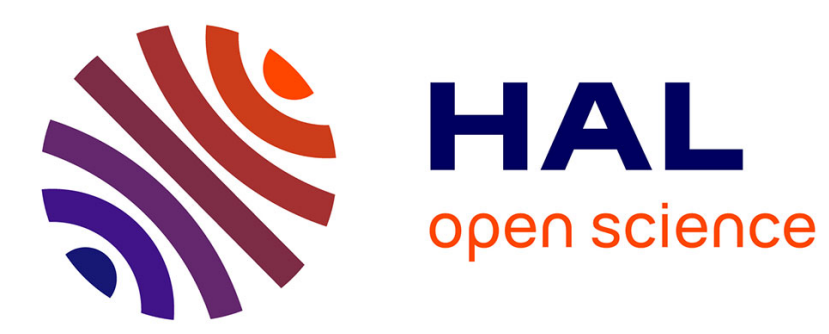

\title{
Coupling FEM and symmetric BEM for dynamic interaction of dam-reservoir systems
}

A. Seghir, A. Tahacourt, Guy Bonnet

\section{To cite this version:}

A. Seghir, A. Tahacourt, Guy Bonnet. Coupling FEM and symmetric BEM for dynamic interaction of dam-reservoir systems. Engineering Analysis with Boundary Elements, 2009, 33 (10), pp.1201-1210. 10.1016/j.enganabound.2009.04.011 . hal-00691013

\section{HAL Id: hal-00691013 https://hal.science/hal-00691013}

Submitted on 18 Jan 2016

HAL is a multi-disciplinary open access archive for the deposit and dissemination of scientific research documents, whether they are published or not. The documents may come from teaching and research institutions in France or abroad, or from public or private research centers.
L'archive ouverte pluridisciplinaire HAL, est destinée au dépôt et à la diffusion de documents scientifiques de niveau recherche, publiés ou non, émanant des établissements d'enseignement et de recherche français ou étrangers, des laboratoires publics ou privés. 


\title{
Coupling FEM and symmetric BEM for dynamic interaction of dam-reservoir systems
}

\author{
A. Seghir ${ }^{\text {a,c }}$, A. Tahakourt ${ }^{\text {b }}$, G. Bonnet ${ }^{\text {c,* }}$ \\ a Université A.Mira de Béjaia, Département de Génie Civil, 06000, Route de Targua Ouzemour, Béjaia, Algeria \\ ' Université A.Mira de Béjaia, Laboratoire de Technologie des Matériaux et de Génie des Procédés, Algeria \\ ${ }^{\mathrm{c}}$ Université Paris-Est, Laboratoire de Modélisation et Simulation Multi-Echelle, FRE 3160, 5, boulevard Descartes, 77454 Marne-la-Vallée, Cedex, France
}

\section{A B S T R A C T}

A numerical model coupling boundary and finite elements suitable for dynamic dam-reservoir interaction is presented herein. This model involves standard finite element idealization of the dam structure displacements and a new symmetric boundary element formulation of the unbounded reservoir domain leading to an equivalent symmetric stiffness matrix for the discretized pressure field. These two basic parts of the computation are directly coupled by imposing an equilibrium condition at the fluid-structure interface, then the resulting algebraic system is reduced by localizing the coupled terms in the global mass matrix such as usually achieved in the added-mass formulation. Finally, the performance and the accuracy of this model are examined by comparing its results to those obtained from three other numerical models.

Keywords:

Symmetric BEM

FEM

Dam

Fluid-structure

Dynamic interaction

Unbounded reservoir

Seismic response

\section{Introduction}

Two key aspects must be taken into account when dealing with the development of a numerical model for a dynamic problem involving fluid-structure interaction such as the seismic response of concrete gravity dams. The first one is related to the inclusion of dissipative effects due to viscous and radiation damping associated with material properties and domain boundaries, respectively. The second one concerns the choice of the basic variables to be retained for the analysis in order to avoid some typical numerically undesirable properties for the resulting algebraic system such as symmetry loss, zeros diagonal terms, ill-conditioned or non-positive definite matrices.

Traditionally, the structural displacements and the fluid hydrodynamic pressures are used as nodal variables in the finite element modelling of fluid-structure systems $[1,2]$. In the case of a compressible fluid, the resulting algebraic system involves nonsymmetric coupled matrices. To overcome this drawback, various symmetrization techniques are proposed, but practically all of them imply either inversion of large matrices or some changes of variables inducing static condensation or zero diagonal handling $[1,3-5]$. Over the available techniques, a performed one $[4,7]$ is recommended which avoids at the same time the change of variable and the matrix inversion process.

* Corresponding author.

E-mail address: guy.bonnet@univ-paris-est.fr (G. Bonnet).
Other ideas make use of two variables to describe the fluid behavior by mixed formulations based on "pressure and displacement-potential" or "pressure and velocity-potential", while many researchers formulated the governing equations of the fluid in terms of displacements only. A description of these models can be found in Ref. [6]. In these cases too, besides increasing the size of the matrices, zero diagonal terms may be introduced and a special care must be taken when coupling with the structural displacement equation.

Furthermore, even with the symmetry problem efficiently solved, the unbounded reservoir domain still remains a serious source of difficulty for the complete finite element modelling of both structure and water. Indeed, it is well known that this method is a very powerful tool for finite domains which may include inhomogeneities or nonlinearities, but when it is applied to unbounded domains, an artificial truncation boundary is unavoidable and some way of handling boundary conditions at infinity becomes necessary. For this purpose, and in order to take into account the radiation damping, several techniques are employed including absorbing elements, radiating boundaries, infinite elements. A lot of works have been done in this field and a wide literature can be found (see e.g. [1,7-13]).

On the other hand, the boundary element method $[14,18]$ is particularly effective for unbounded domains. It is very popular and largely applied in acoustics and dynamic interaction of soil-structure and fluid-structure systems; soil and fluid unbounded mediums involved in these problems can efficiently be handled $[19,20]$. The other well known advantage of this method 
is that it reduces by one the problem dimension. Memory capacity and computational time can thus be saved. However, most of the boundary element analyses are carried out in the frequency domain. Transient solutions are often obtained from the frequency solutions by using Fourier transformations; they are thus restricted to the cases of linear material behavior.

Moreover, in addition to singular integrals induced in this method, the non-symmetric property of the resulting matrices, considered above against complete finite element modelling of both the structure and the fluid domains, is typical and usual in classical collocation boundary element modelling. Several kinds of methods and diverse numerical schemes have been proposed to overcome difficulties related to the problem of singularity [21,22] but for improving the properties of the resulting algebraic system, complete symmetric boundary element formulations are developed particularly for numerical models coupling finite and boundary elements $[23,24]$.

The idea of coupling these two methods is strongly motivated by combining their advantages and reducing their disadvantages. A lot of studies are carried out by using different formulations for the boundary integral part of the problem, such as: Galerkin method [24,25], dual reciprocity boundary elements [26,27] variational principals [28], special Green's functions [29], direct coupling via a transformation matrix [30], discontinuous boundary elements [31], etc. Even though the great part of the coupling techniques enforces one of the two methods to be in the same format as the other to make them compatible, a number of researchers, however, prefer to preserve the nature of both methods by using an interface relaxation algorithm or some other iteration scheme to satisfy the equilibrium condition at the fluid-structure interface $[32,33]$.

In the present paper, an indirect regularized boundary integral formulation [18] is used to discretize the equation of the potential energy supplied by a repartition of potential sources in the fluid domain. An equivalent positive definite and symmetric stiffness matrix is then obtained leading to a linear algebraic system for the pressure variable similar to that of the standard static displacement finite element formulation. This equivalent stiffness matrix for boundary integral formulation is introduced by Bonnet et al. [34]. It looks like a finite element matrix and can easily be assembled or coupled to a finite element model. For the case of the dam-reservoir system under consideration, a direct coupling is achieved by using an interaction matrix linking the pressure and displacement variables. It leads to the wel known added-mass model $[2,35,36]$ for the dam structural displacements.

\section{The dam finite element model}

The finite element discrete system of differential equations describing the dam structure displacements can be written as follows:

$\boldsymbol{M}_{S} \ddot{\boldsymbol{U}}+\boldsymbol{C}_{S} \dot{\boldsymbol{U}}+\boldsymbol{K}_{S} \boldsymbol{U}=\boldsymbol{F}_{\mathrm{g}}+\boldsymbol{F}_{p}$

where $\boldsymbol{M}_{\mathrm{S}}, \boldsymbol{C}_{\mathrm{S}}$ and $\boldsymbol{K}_{\mathrm{S}}$ are the classical mass, damping and stiffness matrices. The unknown vector of basic nodal variables $\boldsymbol{U}$ represents the relative displacements at the nodal points of the finite element model of the dam and the dot symbol (c) denotes differentiation with respect to time $t$.

The global damping matrix, characterizing the energy dissipation within the dam, is most effectively constructed by applying the concept of Rayleigh damping and may be computed from the equation

$\boldsymbol{C}_{S}=\alpha \boldsymbol{M}_{S}+\beta \boldsymbol{K}_{S}$

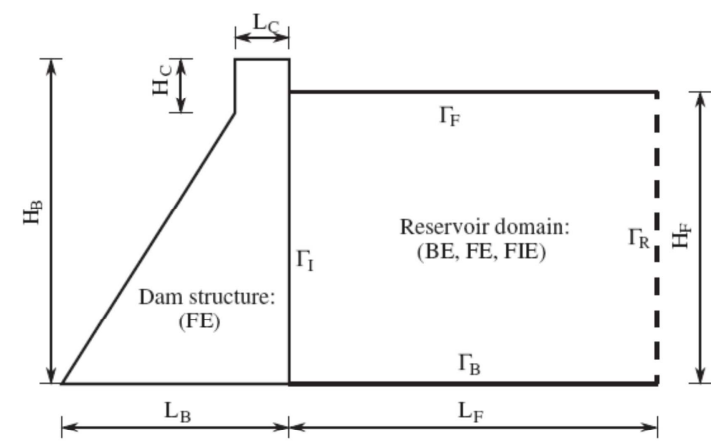

Fig. 1. Dam-reservoir coupled model.

where $\alpha$ and $\beta$ are proportionality constants selected to control the damping of the lowest and highest modes expected to contribute significantly to the response.

The forcing vector

$$
\boldsymbol{F}_{g}=-\boldsymbol{M}_{S} \boldsymbol{I} \ddot{u}_{g}(t)
$$

contains the driving force components generated by the time varying prescribed ground accelerations $\ddot{u}_{g}(t)$ applied at the structure nodal points via the unit influence vector $\boldsymbol{I}$.

The additional forcing vector

$$
\boldsymbol{F}_{p}=\boldsymbol{Q P}
$$

of hydrodynamic forces acting on the upstream face of the dam is related to the unknown vector of nodal pressures $\boldsymbol{P}$ through the transformation matrix $\boldsymbol{Q}$ given by

$$
\boldsymbol{Q}=\int_{\Gamma_{1}} \boldsymbol{N}_{u}^{T} \boldsymbol{n} \boldsymbol{N}_{p} \mathrm{~d} \Gamma
$$

This expression arises from the discretization of the boundary condition

$\sigma_{i j} n_{j}=p n_{i} \quad \mid \Gamma_{\mathrm{l}}$.

which represents the equilibrium of the normal forces acting in the $i$ direction between stress vector and pressure at the fluid-structure interface $\Gamma_{1}$ of unit normal vector $\boldsymbol{n}$ (Fig. 1).

The two vectors $\boldsymbol{N}_{u}$ and $\boldsymbol{N}_{p}$ in Eq. (5) contain shape functions used for the nodal interpolation of the displacement and of the pressure fields, respectively. It is worthwhile to notice that the vector $\boldsymbol{P}$ of the nodal pressures can as well be derived from a finite element model, from a boundary element model, or from other discretizing processes.

\section{The reservoir boundary element model}

3.1. Governing equation and boundary conditions

Under the assumptions of incompressible and inviscid water with a two-dimensional small amplitude motion, it can be shown that the hydrodynamic pressure field $p$ in the reservoir unbounded domain, relative to a Cartesian coordinate system $\left(O x_{1} x_{2}\right)$, complies with Laplace's equation

$\nabla^{2} p=\frac{\partial^{2} p}{\partial x_{1}^{2}}+\frac{\partial^{2} p}{\partial x_{2}^{2}}=0$

with the following boundary conditions:

(i) At the fluid-structure interface $\Gamma_{\mathrm{l}}$, the boundary condition in Eq. (6) is now expressed in terms of equilibrium between 
pressure gradients and normal accelerations

$\frac{\partial p}{\partial \boldsymbol{n}}=-\rho \ddot{\boldsymbol{u}} \cdot \boldsymbol{n}$

where $\rho$ is the mass density of the fluid and $\ddot{\boldsymbol{u}}$ is the acceleration vector of points located at the interface.

(ii) At the reservoir bottom $\Gamma_{\mathrm{B}}$, the same condition as above applies, but in the present case the normal forces are due to the soil. This cancels the pressure gradients for a horizontal bottom surface hypothesis with horizontal ground motion

$$
\frac{\partial p}{\partial \boldsymbol{n}}=0
$$

(iii) At the reservoir free surface $\Gamma_{\mathrm{S}}$, the most common assumption is to set the hydrodynamic pressure at the atmospheric pressure value. This is equivalent to neglecting surface wave effects $[1,2]$

$$
p=0
$$

(iv) At the far away from the upstream face of the dam, the reservoir extends theoretically to infinity. A truncation boundary with an appropriate condition can be used to terminate the infinite domain at some distance intended close to the fluid-structure interface in order to minimize the size of the vector $\boldsymbol{P}$. It is also possible with the boundary element method to leave the domain opened and to mesh only the free surface and the bottom of the reservoir till a certain distance to be evaluated numerically. Therefore, the boundary $\Gamma_{\mathrm{R}}$ in Fig. 1 can simply be removed from the boundary discretization.

It must be pointed out here that because of incompressible water hypothesis, this mathematical description of the reservoir by Laplace's equation does not take into account the propagation effects. The hydrodynamic pressures caused by the motions of the dam structure and computed by using the incompressibility assumption decrease instantaneously along the upstream direction of the reservoir. However, the assumption of incompressible fluid is widely used in the seismic engineering computations involving fluid-structure interaction, because in the main frequency range involved in the seismic motion, the compressibility effects are negligible [15-17].

\subsection{Boundary integrals equations}

The integral equation giving the pressure value $p(\boldsymbol{x})$ at a point $\boldsymbol{x}$ of coordinates $\left(x_{1}, x_{2}\right)$ on the boundary $\partial \Omega_{F}$ delimiting the fluid domain $\Omega_{F}$ can be expressed as [18]

$c(\boldsymbol{x}) p(\boldsymbol{x})=\int_{\partial \Omega_{F}}[q(\boldsymbol{y}) G(\boldsymbol{x}, \boldsymbol{y})-p(\boldsymbol{y}) H(\boldsymbol{x}, \boldsymbol{y})] \mathrm{d} S_{y}$

where $q(\boldsymbol{y})=\partial p(\boldsymbol{y}) / \partial \boldsymbol{n}(\boldsymbol{y})$ is the normal derivative of the pressure $p(\boldsymbol{y})$ at a point $\boldsymbol{y}$ of coordinates $\left(y_{1}, y_{2}\right)$ on the boundary.

The point $\boldsymbol{x}$ denotes the source point and $\boldsymbol{y}$ the current point. Functions $G(\boldsymbol{x}, \boldsymbol{y})$ and $H(\boldsymbol{x}, \boldsymbol{y})$ are the fundamental solution for Laplace's equation (or Green's function) and its normal derivative, respectively. They are usually given as functions of the radius $r(\boldsymbol{x}, \boldsymbol{y})$ between the two points $\boldsymbol{x}$ and $\boldsymbol{y}$ as follows:

$G(\boldsymbol{x}, \boldsymbol{y})=\frac{1}{2 \pi} \ln \frac{1}{r}, \quad H(\boldsymbol{x}, \boldsymbol{y})=\frac{-1}{2 \pi r} \frac{\partial r}{\partial \boldsymbol{n}(\boldsymbol{y})}$

The free term $c(\boldsymbol{x})$ is defined as the following limit when a small surface $S_{\varepsilon}$ of radius $\varepsilon$ surrounding the point $\boldsymbol{x}$ on $\partial \Omega_{F}$ tends to zero:

$C(\boldsymbol{x})=\lim _{\varepsilon \rightarrow 0} \int_{S_{\varepsilon}} H(\boldsymbol{x}, \boldsymbol{y}) \mathrm{d} S_{y}$
It takes the values 1 or 0 depending on whether the point $\boldsymbol{x}$ is within or outside the domain, and $\frac{1}{2}$ if $\boldsymbol{x}$ is on a smooth part of the boundary.

Writing Eq. (11) for the complementary domain $\Omega_{c}=\mathbb{R}^{2}-\Omega_{F}$, where $\mathbb{R}^{2}$ is the Euclidian two-dimensional space, and denoting by $p_{c}$ and $q_{c}$ the solutions of Laplace's equation in $\Omega_{c}$, one can write for a point $\boldsymbol{x}$ within $\Omega_{F}$ :

$p(\boldsymbol{x})=\int_{\partial \Omega_{F}}\left(\left[q(\boldsymbol{y})+q_{c}(\boldsymbol{y})\right] G(\boldsymbol{x}, \boldsymbol{y})-\left[p(\boldsymbol{y})-p_{c}(\boldsymbol{y})\right] H(\boldsymbol{x}, \boldsymbol{y})\right) \mathrm{d} S_{y}$

Taking into consideration the continuity of the pressure field $\left(p=p_{c}\right.$ ) between both sides of the fluid boundary $\partial \Omega_{F}$ and introducing the flux jump:

$\phi(\boldsymbol{y})=q_{c}(\boldsymbol{y})-q(\boldsymbol{y})=-\left[\nabla p_{c}(\boldsymbol{y})-\nabla p(\boldsymbol{y})\right] \cdot \boldsymbol{n}(\boldsymbol{y})$

leads to the integral representation of $p$ by the single layer potential of density $\phi$

$p(\boldsymbol{x})=\int_{\partial \Omega_{F}} \phi(\boldsymbol{y}) G(\boldsymbol{x}, \boldsymbol{y}) \mathrm{d} S_{y}$

and hence, the flux $q(\boldsymbol{x})$ can be obtained by simple derivation with respect to the normal $\boldsymbol{n}(\boldsymbol{x})$

$q(\boldsymbol{x})=\frac{\partial p(\boldsymbol{x})}{\partial \boldsymbol{n}(\boldsymbol{x})}=\int_{\partial \Omega_{\mathrm{F}}} \phi(\boldsymbol{y}) H(\boldsymbol{y}, \boldsymbol{x}) \mathrm{d} S_{y}$

\subsection{Energy boundary integral equation}

The external energy supplied by the flux along the whole boundary can be written as

$W=\int_{\partial \Omega_{\mathrm{F}}} q(\boldsymbol{y}) \cdot p(\boldsymbol{y}) \mathrm{d} S_{y}$

In order to avoid the singular integrals involved in this expression, it is rewritten in the following regularized form (some changes in the variables are also made, see [34] for complete details):

$W=\int_{\partial \Omega_{F}} \phi(\boldsymbol{x}) \int_{\partial \Omega_{F}} H(\boldsymbol{x}, \boldsymbol{y})[p(\boldsymbol{y})-p(\boldsymbol{x})] \mathrm{d} S_{y} \mathrm{~d} S_{x}$

The difference in the pressure values between the two points $\boldsymbol{x}$ and $\boldsymbol{y}$ is given according to Eq. (16) by

$p(\boldsymbol{x})-p(\boldsymbol{y})=\int_{\partial \Omega_{\mathrm{F}}} \phi(\boldsymbol{z})[G(\boldsymbol{z}, \boldsymbol{y})-G(\boldsymbol{z}, \boldsymbol{x})] \mathrm{d} S_{z}$

where $\boldsymbol{z}$ is a dummy variable introduced to represent points of coordinates $\left(z_{1}, z_{2}\right)$ on $\partial \Omega_{F}$.

\subsection{Energy boundary element discretization}

In order to make this boundary element formulation easy for the coupling with the finite element model of the dam structure by matching the corresponding meshes, it is preferred to make use of linear boundary elements rather than constant or higher order ones.

The unknown single layer potential $\phi$ is approximated using nodal interpolation by

$\phi(\boldsymbol{x})=\sum_{i=1}^{n=2} N_{i}(\boldsymbol{x}) \phi_{i} \equiv \boldsymbol{N}_{e_{i}} \phi_{e_{i}}$

where $N_{i}$ are the two shape functions of contour elements and $\phi_{i}$ are the nodal values of potential $\phi$ at point $\boldsymbol{x}_{i}$ within the element $e_{i}$.

Using the subscript $j$ for the discrete values of $\phi_{j}$ at points $\boldsymbol{z}_{j}$ in an element $e_{j}$, the discrete expression of the energy given by the 
two equations (19) and (20) is

$W=\sum_{e_{i}=1}^{n_{e}} \sum_{e_{j}=1}^{n_{e}} \int_{e_{i}} \int_{e_{j}} \boldsymbol{N}_{e_{i}} \phi_{e_{i}} \Im\left(\Omega_{F}\right) \boldsymbol{N}_{e_{j}} \phi_{e_{j}} \mathrm{~d} S_{x} \mathrm{~d} S_{z}$

The summation sign is regarded here as an assembly operator over the $n_{e}$ elements constituting the boundary $\partial \Omega_{F}$ like in the finite element assemblage. The term $\mathfrak{I}\left(\Omega_{F}\right)$ is given by

$\mathfrak{I}\left(\Omega_{F}\right)=\int_{\partial \Omega_{F}} H(\boldsymbol{x}, \boldsymbol{y})[G(\boldsymbol{z}, \boldsymbol{y})-G(\boldsymbol{z}, \boldsymbol{x})] \mathrm{d} S_{y}$

These two last equations lead, for a pair of elements $\left(e_{i}, e_{j}\right)$, to the following expression of the elementary energy $W_{i j}^{(e)}$ :

$W_{i j}^{(e)}=\phi_{j}^{T} A_{i j}^{(e)} \phi_{i}$

where $\boldsymbol{A}_{i j}^{(e)}$ is a $2 \times 2$ element matrix. If each linear element is defined by two nodes named 1 and 2 , this matrix can be explicited as

$$
\begin{aligned}
\boldsymbol{A}_{i j}^{(e)}= & \sum_{k=1}^{n_{e}} \int_{\boldsymbol{x}_{i_{1}}}^{\boldsymbol{x}_{i_{2}}} \int_{\boldsymbol{z}_{j_{1}}}^{\boldsymbol{z}_{j_{2}}} \int_{\boldsymbol{y}_{k_{1}}}^{\boldsymbol{y}_{k_{2}}}\left\langle N_{1}(\boldsymbol{z}) N_{2}(\boldsymbol{z})\right\rangle H(\boldsymbol{x}, \boldsymbol{y}) \\
& \times(G(\boldsymbol{z}, \boldsymbol{y})-G(\boldsymbol{z}, \boldsymbol{x}))\left[\begin{array}{c}
N_{1}(\boldsymbol{x}) \\
N_{2}(\boldsymbol{x})
\end{array}\right] \mathrm{d} S_{y} \mathrm{~d} S_{z} \mathrm{~d} S_{x}
\end{aligned}
$$

Assembling for all elements of the reservoir boundary gives the discrete expression of the total energy

$$
W=\phi^{T} \boldsymbol{A} \phi
$$

It is worthwhile to precise here that even if the element matrix $\boldsymbol{A}_{i j}^{(e)}$ does not appear symmetric, the global assembled matrix $\boldsymbol{A}$ is symmetric due to the equality of the contributions coming from pairs of elements $\left(e_{i}, e_{j}\right)$ and $\left(e_{j}, e_{i}\right)$.

Expressing now the values of the potential density vector $\phi$ by using the discretized form of Eq. (16) as

$\phi=\boldsymbol{G}^{-1} \boldsymbol{P}, \quad \boldsymbol{G}_{i j}=\int_{e_{j}} \boldsymbol{N}^{T} G\left(\boldsymbol{x}_{i}, \boldsymbol{y}\right) \mathrm{d} S_{y}$

and replacing in Eq. (26), the discrete expression of the total energy becomes

$W=\{\boldsymbol{P}\}^{T}\left[\boldsymbol{G}^{-1}\right]^{T}[\boldsymbol{A}]\left[\boldsymbol{G}^{-1}\right]\{\boldsymbol{P}\}$

\subsection{Equivalent stiffness matrix}

By discretizing now Eq. (18) for the total energy $W$ over the entire fluid boundary, the following expression arises:

$$
W=\int_{\partial \Omega_{F}} \boldsymbol{P}^{T} \boldsymbol{N}^{T} q(\boldsymbol{y}) \mathrm{d} S_{y}
$$

Comparing these two later expressions ((28) and (29)), one can write the following equality by suppressing the row vector $\boldsymbol{P}^{T}$ from the left sides:

$\left[\boldsymbol{G}^{-1}\right]^{T}[\boldsymbol{A}]\left[\boldsymbol{G}^{-1}\right] \boldsymbol{P}=\int_{\partial \Omega_{F}} \boldsymbol{N}^{T} q(\boldsymbol{y}) \mathrm{d} S_{y}$

which is an equivalent finite element static equation for the reservoir pressure field:

$\boldsymbol{K}_{F} \boldsymbol{P}=\boldsymbol{F}_{q}, \quad \boldsymbol{K}_{F}=\left[\boldsymbol{G}^{-1}\right]^{T}[\boldsymbol{A}]\left[\boldsymbol{G}^{-1}\right]$

Compared to the standard boundary element formulation, this expression can easily be coupled with a finite element model without symmetry loss. In addition, according to [18], the singularity properties in the boundary integral equations tend to make the resulting matrices diagonally dominant. This is the same situation as in Eq. (25). The above produced algebraic system is consequently well conditioned.

\section{The dam-reservoir coupled model}

In the case of the reservoir under consideration, the force vector $\boldsymbol{F}_{q}$ is due to the accelerations $\ddot{\boldsymbol{u}}$ at the upstream face of the dam. The right-hand side of Eq. (30), associated to the four boundary conditions of the reservoir model (Section 2), gives the expression of the force vector $\boldsymbol{F}_{q}$ as

$\boldsymbol{F}_{q}=-\int_{\Gamma_{1}} \boldsymbol{N}_{p}^{T} \rho \ddot{\boldsymbol{u}} \cdot \boldsymbol{n} \mathrm{d} \Gamma$

Replacing the acceleration vector by its nodal approximation $\ddot{\boldsymbol{u}}=\boldsymbol{N}_{u} \ddot{\boldsymbol{U}}$, the above equation leads exactly to the transposed matrix $\boldsymbol{Q}^{T}$ of Eq. (5) multiplied by the fluid density $\rho$. Hence, Eq. (30) becomes

$\boldsymbol{K}_{F} \boldsymbol{P}=-\rho \boldsymbol{Q}^{T} \ddot{\boldsymbol{U}}$

from which the pressure vector $\boldsymbol{P}$ can be computed

$\boldsymbol{P}=-\rho \boldsymbol{K}_{F}^{-1} \ddot{\boldsymbol{U}}$

and replaced in expression (4) of the hydrodynamic forces $\boldsymbol{F}_{p}$ to give the following dynamic equation for the structure displacements:

$\left[\boldsymbol{M}_{S}+\rho \boldsymbol{Q} \boldsymbol{K}_{F}^{-1} \boldsymbol{Q}^{T}\right] \ddot{\boldsymbol{U}}+\boldsymbol{C}_{S} \dot{\boldsymbol{U}}+\boldsymbol{K}_{S} \boldsymbol{U}=\boldsymbol{F}$

This is the well known added-mass expression usually obtained in finite element modelling of fluid-structure interaction. All matrices here are symmetric and positive definite if the structure is fixed somewhere. The system can thus be handled by the standard finite element solution procedures.

\section{Case study and numerical results}

The performance and the accuracy of the present symmetric boundary element model are demonstrated through the evaluation of the dynamic response of the Pine Flat dam which is extensively analyzed in several previous studies. Ref. [37] is especially used here for comparison. Referring to Fig. 1, the dimensions of the dam-reservoir system are given in Table 1 with the material properties of the dam concrete, where $E$ is Young's modulus in $10^{9} \mathrm{~N} / \mathrm{m}^{2}, \rho$ is the mass density in $\mathrm{kg} / \mathrm{m}^{3}$ and $v$ is Poisson's ratio.

The horizontal length $L_{\mathrm{F}}$ of the reservoir domain corresponds to the position of the truncation boundary which is fixed for a first analysis at three times the total height $H_{\mathrm{B}}$ of the dam. This position is shown, in several previous studies, to be far enough from the fluid-structure interface to make unnecessary the use of a radiating boundary. After that, it is moved closer to the interface in order to assess the position at which the numerical models used here still remain effective. Results corresponding to $L_{\mathrm{F}}=$ $0.25 H_{\mathrm{B}}$ are reported hereafter as an example of a close position.

In all the parametric study conducted in this work, the dam structure is modelled by four-nodes linear elements of element size fixed approximatively to $5 \mathrm{~m}$. This gives a dam mesh that consists in a total of 600 elements and 651 nodes resulting in 1260

\begin{tabular}{|c|c|c|c|c|c|c|c|c|}
\hline \multicolumn{6}{|c|}{ Dimensions (m) } & \multicolumn{3}{|c|}{ Material properties } \\
\hline$H_{\mathrm{B}}$ & $H_{\mathrm{C}}$ & $L_{\mathrm{B}}$ & $L_{C}$ & $L_{\mathrm{F}}$ & $H_{\mathrm{F}}$ & $E$ & $v$ & $\rho$ \\
\hline 122.0 & 18.5 & 96.0 & 9.75 & 366.0 & 116.0 & 34.47 & 0.2 & 2440 \\
\hline
\end{tabular}
degrees of freedom (DOFs). Whereas, the unbounded reservoir

Table 1

Geometry and material properties of the Pine Flat dam. 
domain is idealized in four different ways leading to the four following coupling models for the entire dam-reservoir system:

(1) FE-AM: the reservoir is represented by the added-mass concept using Westergaard's simplified approach [38]. In this model, an equivalent concrete mass of parabolic shape representing the part of the reservoir affected by the vibrations of the dam structure is added to the mass matrix of the finite element equation (1). The expression of this parabolic shape is $\frac{7}{8} \sqrt{H_{\mathrm{F}}\left(H_{\mathrm{F}}-y\right)}$ where $y$ is the vertical coordinate along the upstream face of the dam measured from the bottom. It is clear that this model does not take explicitly into account the fluid-structure interaction.

(2) FE-FE: the reservoir is modelled by finite elements in the same manner as the dam, but with one degree of freedom per node representing the pressure. No boundary conditions are used to handle the truncation effects. The resulting system of finite element equations is the same as Eq. (35) but the matrix $\boldsymbol{K}_{F}$ is constructed following the finite element discretization of Laplace's equation.

(3) FE-IE: here the finite part of the reservoir is modelled by using finite elements like in the previous model, but the infinite truncated part is represented by appropriate infinite elements based on the direct approach with exponentially decaying shape functions. Details of this model and its application to a dam-reservoir system can be found in Refs. [7,13].

(4) FE-BE: in this model, the reservoir is modelled by the present symmetric boundary element formulation as described above. Linear two-nodes elements are used to discretize the reservoi boundary. The infinite part of the reservoir is simply handled by leaving the geometry opened at the upstream side.

In the last three numerical models, the height of the fluid elements is taken exactly equal to the height of the solid ones in order to easily match the corresponding meshes at the fluidstructure interface. The submodels are directly coupled by using the continuity condition.

For $L_{\mathrm{F}}=3 \mathrm{H}_{\mathrm{B}}$, the finite element model of the reservoir domain consists in 925 elements and 988 nodes. This gives 950 DOFs afte the boundary condition is applied. This number of elements is reduced to 125 when $L_{\mathrm{F}}=0.25 \mathrm{H}_{\mathrm{B}}$. The unbounded truncated part is represented in FE-IE model by 25 infinite four-nodes elements adding a supplement of 52 DOFs to the model. The FE-BE mode consists only in 100 elements when $L_{\mathrm{F}}=3 H_{\mathrm{B}}$ which gives 62 DOFs. This is reduced to 34 elements and 28 DOFs when $L_{\mathrm{F}}=0.25 H_{\mathrm{B}}$.

\subsection{Modal analysis}

Modal analysis of the dam alone and of the dam-reservoi coupled system are firstly performed to obtain the free vibration modes by solving Eqs. (1) and (35) without the forcing vectors and the dissipative matrices. Table 2 summarizes results obtained for periods of the first five free vibration modes using the four above described numerical models and for the two cases of the reservoi truncation boundary position.

It can be clearly noticed from this table that when the reservoir domain is truncated at a distance $L_{\mathrm{F}}=3 \mathrm{H}_{\mathrm{B}}$, the three variants of the numerical models (FE-FE, FE-IE and FE-BE) yield practically identical values of periods for the complete range of vibration modes retained in the analysis. In addition, these results do not differ sensibly from those obtained by applying the simplified Westergaard's added-mass approach. However, when the reservoir truncation boundary is moved close to the dam-reservoi interface $\left(L_{\mathrm{F}}=0.25 \mathrm{H}_{\mathrm{B}}\right)$, results obtained by the numerical model
Table 2

Periods (s) of the dam alone and of the dam-reservoir system.

\begin{tabular}{|c|c|c|c|c|c|}
\hline Mode number & 1 & 2 & 3 & 4 & 5 \\
\hline \multicolumn{6}{|l|}{ Dam alone } \\
\hline $\mathrm{FE}$ & 0.2558 & 0.1241 & 0.0921 & 0.0705 & 0.0466 \\
\hline \multicolumn{6}{|c|}{ Dam with added-mass approach } \\
\hline FE-AM & 0.2943 & 0.1415 & 0.0934 & 0.0837 & 0.0571 \\
\hline \multicolumn{6}{|c|}{ Dam-reservoir system with numerical models $\left(L_{\mathrm{F}}=3 \mathrm{H}_{\mathrm{B}}\right)$} \\
\hline FE-FE & 0.2810 & 0.1375 & 0.0930 & 0.0762 & 0.0486 \\
\hline FE-IE & 0.2807 & 0.1372 & 0.0929 & 0.0762 & 0.0485 \\
\hline FE-BE & 0.2806 & 0.1385 & 0.0930 & 0.0766 & 0.0487 \\
\hline \multicolumn{6}{|c|}{ Dam-reservoir system with numerical models $\left(L_{\mathrm{F}}=0.25 \mathrm{H}_{\mathrm{B}}\right)$} \\
\hline FE-FE & 0.3073 & 0.1520 & 0.0937 & 0.0798 & 0.0508 \\
\hline FE-IE & 0.2785 & 0.1353 & 0.0928 & 0.0758 & 0.0484 \\
\hline FE-BE & 0.2730 & 0.1313 & 0.0927 & 0.0832 & 0.0523 \\
\hline
\end{tabular}

Table 3

Modal mass participation ratios (\%).

\begin{tabular}{|c|c|c|c|c|c|c|}
\hline Mode number & 1 & 2 & 3 & 4 & 5 & Cumul. \\
\hline \multicolumn{7}{|l|}{ Dam alone } \\
\hline FE & 35.61 & 25.42 & 11.46 & 9.75 & 8.30 & 90.52 \\
\hline \multicolumn{7}{|c|}{ Dam with added-mass approach } \\
\hline FE-AM & 42.30 & 25.92 & 8.69 & 7.64 & 5.61 & 90.17 \\
\hline \multicolumn{7}{|c|}{ Dam-reservoir system with numerical models $\left(L_{\mathrm{F}}=3 \mathrm{H}_{\mathrm{B}}\right)$} \\
\hline FE-FE & 45.33 & 26.21 & 7.01 & 8.70 & 5.56 & 92.81 \\
\hline FE-IE & 45.31 & 26.20 & 6.99 & 8.67 & 5.57 & 92.75 \\
\hline $\mathrm{FE}-\mathrm{BE}$ & 45.82 & 26.43 & 6.91 & 8.41 & 5.26 & 92.82 \\
\hline \multicolumn{7}{|c|}{ Dam-reservoir system with numerical models $\left(L_{\mathrm{F}}=0.25 \mathrm{H}_{\mathrm{B}}\right)$} \\
\hline FE-FE & 57.95 & 24.17 & 5.12 & 4.58 & 1.80 & 93.62 \\
\hline FE-IE & 43.85 & 25.86 & 7.27 & 9.35 & 6.14 & 92.47 \\
\hline FE-BE & 32.09 & 23.74 & 10.95 & 14.32 & 8.41 & 89.52 \\
\hline
\end{tabular}

coupling dam finite elements and reservoir finite elements (FE-FE) diverge somewhat from those obtained when infinite elements are coupled to the reservoir finite elements (FE-FI) or when the reservoir is formulated differently with the present boundary element technique (FE-BE). These two last models lead practically to the same results without quality loss compared to the case of $L_{\mathrm{F}}=3 \mathrm{H}_{\mathrm{B}}$

Overall, these results agree with those given in [37] for the first three vibration periods of the dam alone $(0.2530,0.1223$, $0.0907 \mathrm{~s}$.) and of the dam-reservoir system $(0.2980,0.1372$, $0.0915 \mathrm{~s}$.). The authors in [37] simulated the Pine Flat dam-reservoir system by a complete finite element model using both Eulerian and Lagrangian approaches for the reservoir subdomain.

In addition, complementary modal analysis results are given in Table 3 where are reported the modal mass participation ratios of the first five vibration modes. The cumulative contributions of the whole five modes are also included for each model.

In this case too, a good agreement between the results of the three numerical models can be noticed when $L_{\mathrm{F}}=3 H_{\mathrm{B}}$. Most important, the total contributions of modal mass participation ratios of the five modes show that the added mass does not affect the dam behavior in the same way as does the fluid-structure interaction. The increasing in the cumulative modal mass ratios seems due to a larger contribution of the five modes when considering the interaction between the two subsystems rather than reducing the reservoir effects to a simple dead mass added to the dam structure.

When the reservoir truncation boundary is positioned close to the fluid-structure interface, the four models lead to differen values of mass participation ratios especially for the first mode. 
Only the model which uses coupling finite and infinite elements for the reservoir does not alter the values of the far position of the truncation boundary. Contrarily to FE-FE, FE-BE tends to increase the modal mass contribution of the higher modes and to decrease the contribution of the first ones. Concerning the cumulative contribution, it is shown that it is not as affected by the position of the truncation boundary as do the modes themselves.

On this basis, it can be concluded that the symmetric boundary element formulation presented in this work does not only allow to derive an equivalent finite element stiffness matrix very attractive for coupling finite and boundary elements, but also is an accurate tool for assessing the dam-reservoir modal analysis. It provides the same results as those of models based on finite elements.

From a numerical point of view, the efficiency of this new technique compared to classical boundary element methods consists essentially in its flexibility to be coupled with finite elements, besides the fact that it does not need to compute hypersingular integrals.

Compared to finite elements, the method can make large computer's memory saving due to the mesh reduction. The main supplementary cost in the construction of the stiffness matrix by the symmetric BEM is due to a computation of multiple integrals, similarly to SGBEM. However, this construction time can be drastically reduced by using parallelized computation of matrix elements with widely used multiprocessors.

The gain on computation time may be assessed for example during the computation of the modal results. The computation time is reduced from 1.02 to $0.42 \mathrm{~s}$ when passing from FEM to BEM. Another difference lies in the computation of the inverse of the stiffness matrix, which is useful during the process (see Eqs. (34) and (35)). The finite element matrix is banded, but its inverse is full and its size is around 15 times the size of the BEM stiffness matrix.

\subsection{Frequency response analysis}

The accuracy and the performance of the present boundary element formulation applied to the reservoir unbounded domain can furthermore be examined by evaluating the frequency response of the dam-reservoir system.

For a ground harmonic excitation $\ddot{u}_{g}(t)=e^{i \omega t}$ of unit acceleration and varying frequency $\omega$ in the range of $0-200 \mathrm{rad} / \mathrm{s}$, Eq. (35) is solved to get harmonic dam nodal displacements $\boldsymbol{U}=\boldsymbol{U}_{\max } e^{i \omega t}$ by using a damping ratio $\xi=5 \%$.

The nodal acceleration vector $\ddot{\boldsymbol{U}}_{\max }=-\omega^{2} \boldsymbol{U}_{\max }$ is then computed and used to evaluate the pressure vector $\boldsymbol{P}$ following Eq. (34) in which the matrix $\boldsymbol{K}_{F}$ must correspond to the model used for the reservoir as indicated in the above description of the four considered models. In the case of the added-mass model, the pressure is not computed because its closed-form expression (Westergaard's formula $[6,38])$ is based on the assumption of a rigid dam.

Figs. 2 and 3 represent the maximum dam horizontal displacements $u$ and accelerations $\ddot{u}$, respectively, and Fig. 4 shows the corresponding maximum hydrodynamic pressures acting at the bottom of the dam expressed as a ratio of the maximum hydrostatic pressures $C_{p}=p /\left(\rho g H_{\mathrm{F}}\right)$.

In this analysis too, it is seen that when the reservoir truncation boundary is placed at $L_{\mathrm{F}}=3 \mathrm{H}_{\mathrm{B}}$, the three numerical models give, for all range of the excitation frequencies, the same results for the dam response and hydrodynamic pressures. When the truncation boundary is moved to $L_{\mathrm{F}}=0.25 \mathrm{H}_{\mathrm{B}}$, the results become different, and especially, inaccurate pressures can be computed by using the FE-FE model due to substantial amplifications caused by lack of a transmitting boundary condition. In addition, it can also be seen that unlike accelerations and pressures, displacements are less affected by the position of the truncation boundary.

b
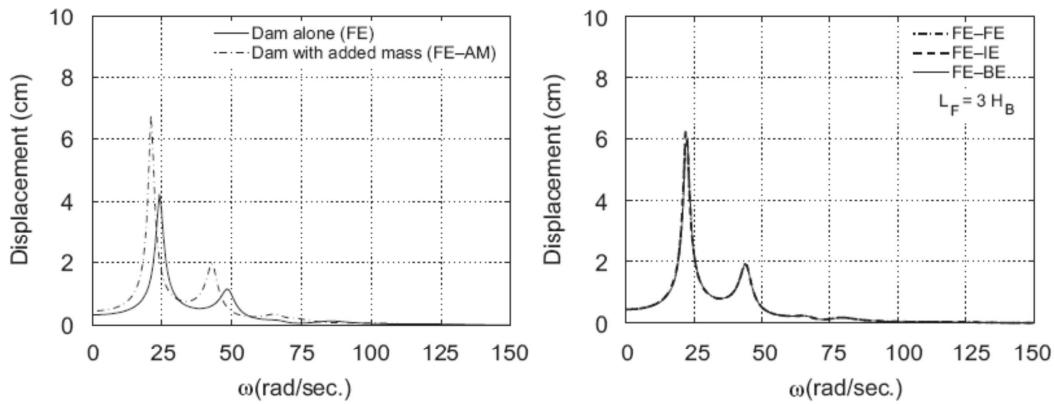

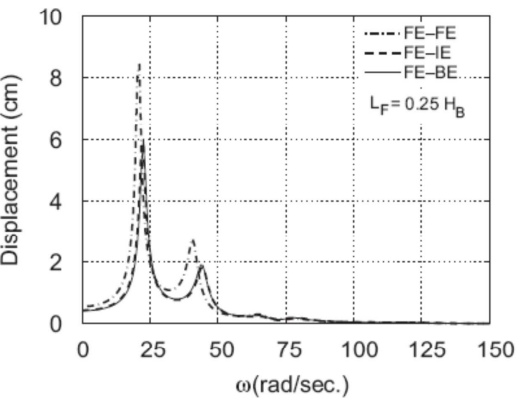

Fig. 2. Frequency response of the maximum dam displacements. 
a

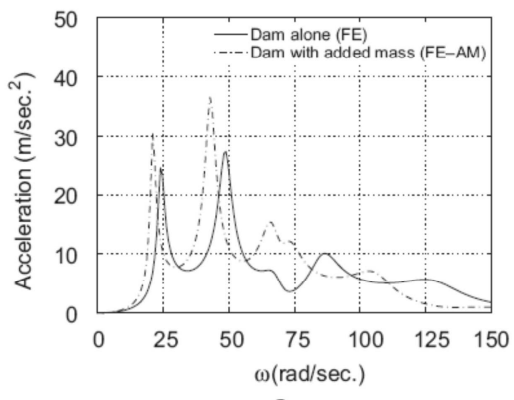

b

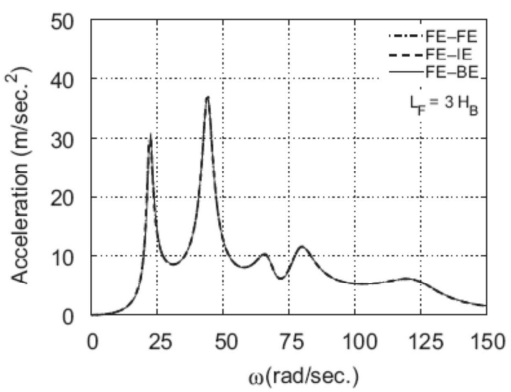

C

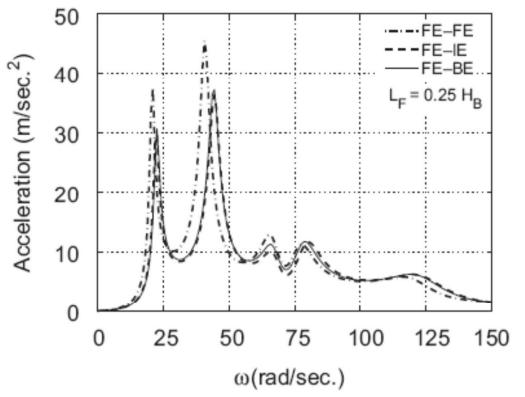

Fig. 3. Frequency response of the maximum dam accelerations.

a

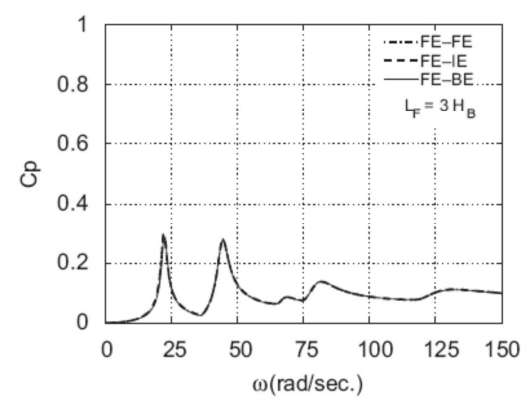

b

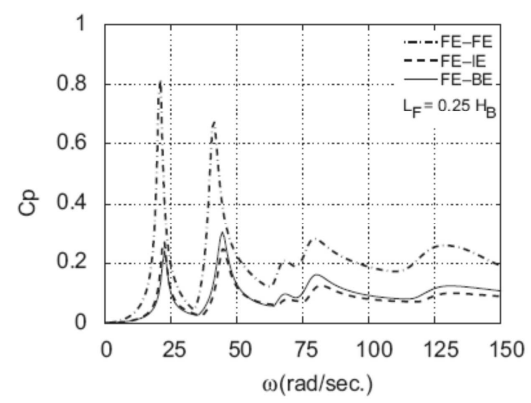

Fig. 4. Frequency response the maximum hydrodynamic pressures.

\subsection{Time history analysis}

Finally, a time history analysis of the dam with and without reservoir effect is performed using the four numerical models described above in order to get more information on the accuracy of the present symmetric boundary element formulation. Recorded horizontal components of the ground accelerations during the Taft earthquake (S69E) shown in Fig. 5 are used in the analysis. Only the first $10 \mathrm{~s}$, which appear to be critical for the response of the dam-water system, and that can show plainly the differences between the results, are considered. The solutions are computed using the modal superposition method with 30 modes and the same damping ratio $\xi=5 \%$ is used for all the modes. The computation produced the time histories of the dam nodal displacements and of the reservoir nodal pressures. The results are reported for the dam crest and bottom.

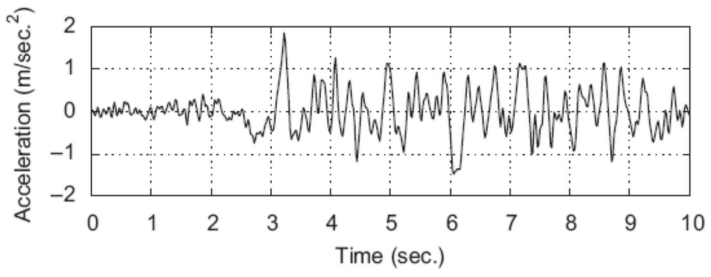

Fig. 5. Horizontal ground acceleration record of the Taft earthquake.

Figs. $6 \mathrm{a}$ and $\mathrm{b}$ show the time history response of the dam crest displacements and accelerations, respectively, obtained for the dam alone (FE model) and for the dam with the reservoir added mass (FE-AM model). 

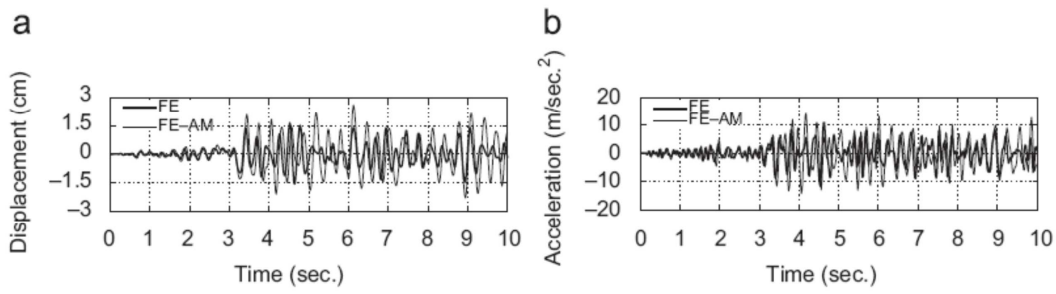

Fig. 6. Time history response of the horizontal displacement and acceleration at the dam crest (dam alone: FE, dam with added mass: FE-AM).
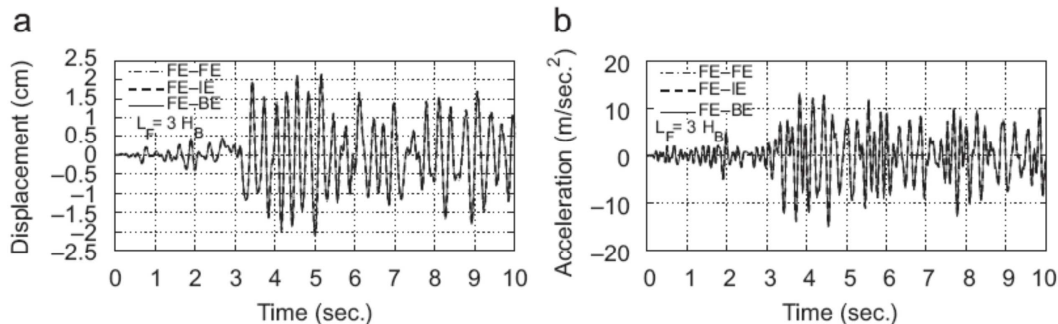

Fig. 7. Time history response of the dam crest horizontal displacement and acceleration (dam-reservoir system with numerical models, $L_{\mathrm{F}}=3 H_{\mathrm{B}}$ ).
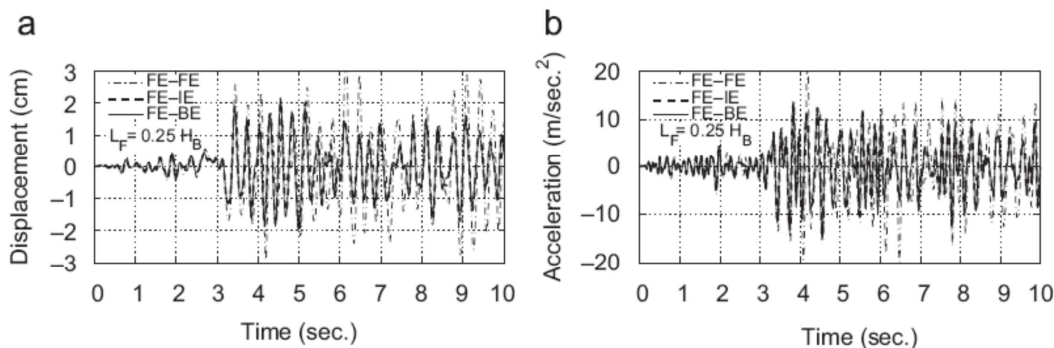

Fig. 8. Time history response of the dam crest horizontal displacement and acceleration (dam-reservoir system with numerical models, $L_{\mathrm{F}}=0.25 H_{\mathrm{B}}$ ).
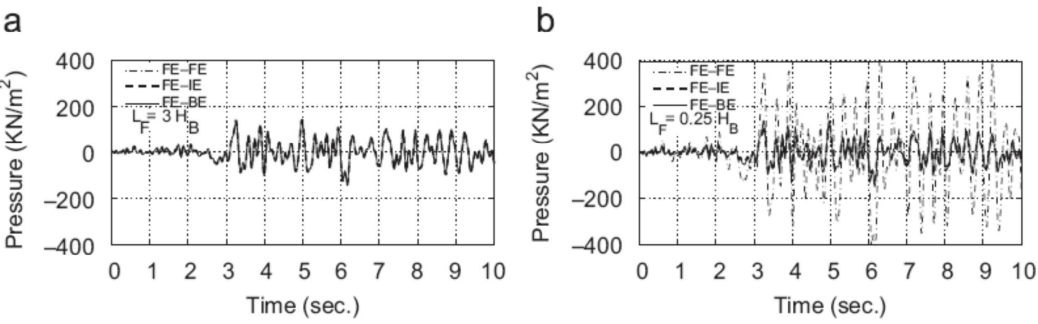

Fig. 9. Time history response of the hydrodynamic pressures acting at the bottom of the upstream face of the dam

It can be seen from this figures that the response of the dam, mainly in terms of displacements, is modified by the added mass. Some amplifications in the peak values and a lowering of the response frequency content can be noticed.

Similar results (Figs. 7a and b) are obtained when the dynamic dam-reservoir interaction is taken into account by applying the three numerical coupling models for the case of a far position of the reservoir truncation boundary $\left(L_{\mathrm{F}}=3 \mathrm{H}_{\mathrm{B}}\right)$. These results show again how the three numerical models are closely equivalent when the mesh of the reservoir is large enough to reduce the effects of the domain truncation.

However in Figs. 8a and b, where results are obtained for the case of a close position of the truncation boundary $\left(L_{\mathrm{F}}=0.25 \mathrm{H}_{\mathrm{B}}\right)$, the discordance between the FE-FE model and the two other models (FE-IE and FE-BE) is clearly apparent. It is to recall that no transmitting boundary condition is used in this model (FE-FE) in order to examine the capability, against infinite elements, of the present symmetric boundary element 
a

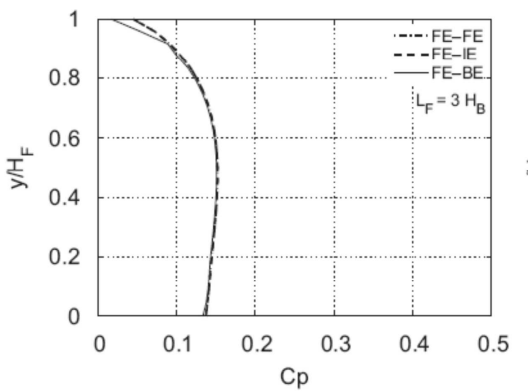

b

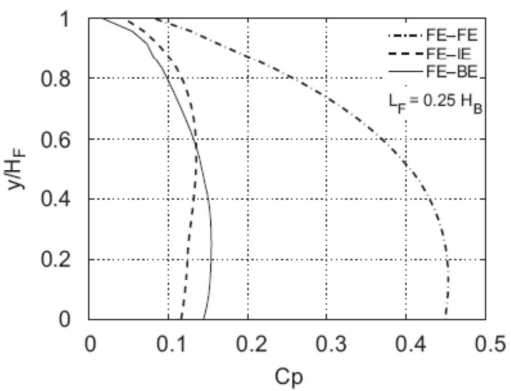

Fig. 10. Hydrodynamic pressure envelope acting on the upstream face of the dam.

formulation to handle the unbounded part of the reservoir domain.

Furthermore, time history response of the pressures acting at the bottom of the upstream face of the dam is evaluated for the two positions of the reservoir truncation boundary and displayed in Fig. 9. It is clearly shown here that the differences between the solutions derived from the FE-FE model and those derived from the two other models are significant. In addition, it appears that the reservoir pressures are largely more sensitive to the applied model than the dam displacements and accelerations. This observation can also be pointed out from the envelop of the pressures acting along the depth on the upstream face of the dam as shown in Fig. 10, where the distribution of hydrodynamic pressures at the dam-water interface is normalized by the maximum hydrostatic pressure $\left(\rho g H_{\mathrm{F}}\right)$ and represented versus the reservoir height ratio $\left(y / H_{\mathrm{F}}\right)$.

\section{Conclusion}

In this study, a numerical model suitable for the evaluation of the dynamic response of concrete gravity dams including fluid-structure interaction has been introduced. It involves a standard finite element modelling of the dam structure and the idealization of the unbounded reservoir domain by a special symmetric boundary integral formulation.

The finite and boundary elements coupling is here greatly simplified by the nature of the resulting boundary element matrix which is equivalent to the finite element stiffness matrix. It is symmetric and can easily be assembled, coupled or subjected to any other finite element treatment.

Results obtained by the application of this numerical model to evaluate the dynamic response of the Pine Flat concrete gravity dam-reservoir system have clearly shown that the unbounded reservoir domain is effectively idealized without any treatment of the infinite part. The model produced results similar to those of a complete finite element modelling of both the dam and the reservoir subdomains.

The agreement between the two kinds of models is reinforced by taking a large reservoir mesh. However, when the reservoir truncation boundary is placed at a distance close to the fluid-structure interface, the complete finite element model fails particularly in evaluating hydrodynamic pressures while the present boundary element formulation still produces accurate results.

\section{References}

[1] Zienkiewicz OC, Taylor RL. The finite element method. 5th ed. Oxford: Butterworth-Heinensann; 2000
[2] Tiliouine B, Seghir A. Fluid-structure models for dynamic studies of damwater systems. In: Eleventh European conference on earthquake engineering 1998, September 6th-11th, Paris, France, 1998.

[3] Daniel WJT. Modal methods in finite element fluid-structure eigenvalue problems. International Journal of Numerical Methods and Engineering 1980;15:1161-75.

4] Tiliouine B, Seghir A. Sur la symétrisation du problème de vibrations des systèmes couplés fluide-structure. In: Cinquième Colloque de l'Association Franç aise de Génie Parasismique 1999, 19-21 Octobre, Paris-Cachan, France, 1999.

[5] Ohayon R. Vibration of fluid-structure coupled systems. In: Oñate E, Periaux J. Samuelson A, editors. Finite Elements in 90's. Barcelona: Springer/CIMNE: 1991.

6] Maity D, Bhattacharyya SK. A parametric study on fluid-structure interaction problems. Journal of Sound and Vibration 2003;263:917-35.

[7] Tiliouine B, Seghir A. A numerical model for time domain analysis of dams including fluid-structure interaction. In: Fourth international conference on computer structures technology 1998, August 18th-20th, Edinburgh, Scotland, 1998.

[8] Bettes P. Infinite elements. UK: Penshaw Press; 1992.

[9] Angelov TA. Infinite elements - theory and applications. Computers \& Structures 1991;41(5):959-62.

[10] Liu DS, Chiou DY. 2-D infinite element modeling for elastostatic problems with geometric singularity and unbounded domain. Computers \& Structures 2005;83:2086-99.

[11] Parrinello F, Borino G. Lagrangian finite element modeling of dam-fluid interaction: accurate absorbing boundary condition. Computers \& Structures 2007;58:932-43.

[12] Abdel-Fattah TT, Hodhod HA, Akl AY. A novel formulation of infinite elements for static analysis. Computers \& Structures 2000;77:371-9.

[13] Seghir A, Tahakourt A. Analyse dynamique des systèmes barrage-reservoir avec couplage éléments finis - éléments infinis. In: Septième Colloque de l'Association Française de Génie Parasismique 2007; 04-06 Juillet, Paris, France, 2007.

[14] Brebbia CA, Dominguez J. Boundary elements: an introductory course Southampton: Computational Mechanics Publications: 1998.

[15] Lin G, Du J, Hu Z. Earthquake analysis of arch and gravity dams including the effects of foundation inhomogeneity, Frontiers of Architectural and Civil Engineering in China 2007:1(1):41-50.

[16] Küçükarslan S. An exact truncation boundary condition for incompressibleunbounded infinite fluid domains. Applied Mathematics and Computation 2005:163:61-9.

[17] Maity D. A novel far-boundary condition for the finite element analysis of infinite reservoir. Applied Mathematics and Computation 2005:170:1314-28. 1 Bonnet M. Equations intégrales et éléments de frontière Applications en mécanique des solides et des fluides. Paris: CNRS Editions/Editions Eyrolles; 1995. 1 Medina F, Domínquez J. Boundary elements for the analysis of the seismic Medina F, Dominquez J. Boundary elements for the analysis of the seismic response of dams including dam-water-foundatio
Analysis with Boundary Elements 1989:6:152-63.

[20] Soares DJ. Mansur W]. Dynamic analysis of fluid-soil-structure interaction problems by boundary Dynamic analysis of fluid-soil-structure interaction problems by boun

[21] Young DL, Hwang WS, Tsai CC, Lu HL. Accuracy of desingularized boundary integral equations for plane exterior potential problems. Engineering Analysis integral equations for plane exterior potent

[22] Zhang X. Zhang X. Exact integrations of two-dimensional high-order discontinuous boundary elements of elastodynamics problems. Engineering Analysis with Boundary Elements 2004:28:725-32.

[23] Gaul L, Wenzel W. A coupled symmetric BE-FE method for acoustic fluid-structure interaction. Engineering Analysis with Boundary Elements 2002;26:629-36

[24] Lehmann L, Antes H. Dynamic structure-soil-structure interaction applying the symmetric Galerkin boundary element method (SGBEM). Mechanics Research Communications 2001;28(3):297-304 
[25] Panzeca T, Cucco F, Terravecchia S. Boundary discretization based on the residual energy using the SGBEM. International Journal of Solids an Structures 2007:44:7239-60.

[26] Chien CC, Chen YH, Chuang CC. Dual reciprocity BEM analysis of 2D transient elastodynamic problems by time-discontinuous Galerkin FEM. Engineerin Analysis with Boundary Elements 2003,27:611-24.

27] Kuçukarslan S. Dynamic analysis of dam-reservoir-foundation interaction in time domain. Computational Mechanics 2004;33:274-81.

ement-boundary integral coupling method for fluid-structure interface. Engineering Analysis with Boundary Elements 1995;15:79-91.

[29] Tao J, Yuying H, Qin Q. A mixed FE-BE method for coupled vibration of gravity dam-reservoir system with variable water depth. Acta Mechanica Solid 2): $123-32$

[30] Yazdchi M, Khalili N, Valliappan S. Dynamic soil-structure interaction analysis via coupled finite-element-boundary-element method. Soil Dy namics and Earthquake Engineering 1999:18:499-517.

[31] Zhang X. Zhang X Coupling FEM and disconting fluid- $X$ tructure interation. Engineering Analysis with Boundary Eles and 2002;26:719-25.
[32] Elleithy WM, Tanaka M. An interface relaxation algorithm for coupling the finite element and boundary element methods. Transactions of JASCOME

2002:2: Paper No. 02-070319.
[33] von Estorff V, Hagen C. Iterative coupling of FEM and BEM in 3D transient elastodynamics. Engineering Analysis with Boundary Elements 2006;30:

[34] Bonnet G, Seghir A, Gorfdir A. Coupling BEM with FEM by a direct computation of the boundary stiffness matrix. Computer Methods in Applied Mechanics and Engineering 2009;198:2439-45.

Rodríguez R, Santamarina D. A finite element solution of an dded mass formulation for fluid-solid vibrations. Numerische Mathematik

[36] Cho JR, Song JM. Assessment of classical numerical models for the separate fluid-structure modal analysis. Journal of Sound and Vibration 2001:239(5): 995-1012.

[37] Calayir Y, Dumanoğlu AA, Bayraktar A. Earthquake analysis of gravity dam-reservoir systems using Eulerian and Lagrangian approaches. Compu-

[38] Westergaard HM. Water pressures on dams during earthquakes. Transactions ASCE 1933:98:1303-18. 